\title{
Destiny or Free Will Decision? A Life Overview from the Perspective of an Informational Modeling of Consciousness Part II: Attitude and Decision Criteria, Free Will and Destiny
}

\author{
Florin Gaiseanu* \\ Principal Researcher, Science and Technology of Information, Romania \\ *Corresponding author: Florin Gaiseanu, Principal Researcher (Professor), Science and Technology of Information, Bucharest, Romania, \\ Email: fgtext@yahoo.es
}

Submission: 此 October 1, 2018; Published: 阱 October 25, 2018

\begin{abstract}
As it was shown in the Part I of this work, the driving of our life is determined by series of YES/NO - type elemental decision, which is actually the information unit (Bit), so we operate actually in an informational mode. The informational analysis and modeling of consciousness reveals seven informational systems, reflected at the conscious level by the cognitive informational centers suggestively called Iknow (Ik - memory), Iwant (Iw decision center), Iove (Il-emotions), Iam (Ia-body status), Icreate (Ic-informational genetic transmitter and educator), Icreated (I-cd genetic generator inherited from the parents) and Ibelieve, (Ib - connection to the informational field of the universe). As the mind can operate in a bipolar mode, we dispose of two alternatives to use our free will: in a positive (YES) or negative (NO) mode. Such modes can be applied as a function of the personal criteria, especially that inhered from the family, which are the fundamental ones, but also from that acquired during the life.

The persistence to operate in a positive or a negative mode is fundamental for the designing of our personal trajectory and therefore for our destiny. The destiny and the role of our free will in deciding the course of our own life are analyzed therefore with respect to our YES/NO attitude, which is an integrated informational output composed by the contribution of all cognition centers, showing that when this is favorable to the life rules, it is avoided its deterioration determined by a permanently negative attitude and thinking mode. It is shown that our intervention should be directed both to the operating mode but also to the decision criteria, when it is ascertained that they do not correspond to the reality, allowing a favorable adaptation. As parents and therefore as forming and educator of the next generation, an activity specific for the center Ic, it is necessary to observe, discover and encourage the talents and predispositions of their Children, to put them on a privileged way of the destiny, because they will benefit of an advanced informational "budget" with respect to their generation and a certain sort of preparation. The comparative analysis between personal operating mode and the reality for adaptation necessities should guide permanently the selected trajectory way, to include new criteria, to change the older, or to reprogramming the operation mode, with the participation of all centers. The stereotype thinking chains triggered by repetitive negative thoughts acts on the informational system as stressing agents, leading to chronic, sometime serious disorders. Such thinking operation forms should be therefore detected and substituted or eliminated without delay, according to some recommended suggestions. Furthermore, it is shown that a specific self-control of the thoughts as positive or negative information and of the lifestyle, helps the health maintenance and the life prolongation under high qualitative conditions.
\end{abstract}

\section{Introduction}

In the Part I of this work we have presented the specific physics and informational arguments [1-3], showing that our informational system operates in a bipolar YES/NO mode and this can be described by seven informational subsystems defined as: Center of Acquisition and Storing of Information (CASI), reflected at the conscious level as a center suggestively called Iknow (abbreviated as Ik), consisting practically in memory and associated external and internal sensors; Center of Decision and Command (CDC), detected at the conscious level as Iwant (Iw), expressing practically the attitude, as a adaptation informational output; the Info-Emotional Center (IES), corresponding to Ilove (Il); Maintenance Informational System (MIS), sustaining the automatic matter-related processing, which corresponds to Iam (Ia); Genetic Transmission System (GTS) reflected in Icreate (Ic) and the Info-Genetic Generator (IGG) cor responding to Icreated (Icd), carrying the info-genetic information from parents. The Anti-entropic Connection (AC) represents the info-relation with dark matter, which is believed to have antisymmetric properties vs. matter properties, specifically antigravity [4], anti-entropy [1,5] and time arrow reversely oriented, from future to present [6]. This connection is detected at the conscious level as the center Ibelieve (Ib), expressing the trust and life confidence. The AC gate allowed to explain [2] the phenomena associated to the Near-Death Experiences (NDE) [7] and to discuss a possible "after-life "existence of consciousness [8] and immortality [9].

On the basis of the informational modeling of consciousness [10] presented in the Part I of this work and the obtained results 
concerning the cognitive centers, in this Part II are discussed the consequences of the bipolar operating YES/NO mode of our decisional system on our life trajectory and destiny. It is shown that the positioning of our free will on the positive (YES) side, according to the requirements of the life prerogatives, we have more chances to benefit of a satisfactory life and destiny. The mechanisms of a repetitive negative operating mode are analyzed, pointing out the emerging processes inducing sometimes some serious and chronic disorders, not only concerning the informational system itself (brain and the nervous cells), but also the other vital organs and systems of our body, like heart, immunity, digestive and sexual systems, leading to a premature aging and shorter life duration.

The definition and understanding of the functions of the informational centers allow observations and conclusions on how we can live our lives optimally, accordingly to its laws and not outside of them, contributing in such manner through our decisions, by the self and lifestyle control, to a harmonious collaboration with our body and with the environment and the society where we live in, for a successful life, aging and destiny.

\section{The attitude as a bipolar informational operator and the decision criteria}

As it was shown in the Part I of this article, our life is driven by YES/NO type decisions. The CDC informational system reflected at the conscious level by Iw, mainly acts from this point of view as an informational operator, influencing or even determining continuously the generation of our trajectory in the life. However, in order to decide, it is necessary to dispose of decision criteria, allowing a distinction between the "Good" and the "Bad". The selection is thus a binary, informational system. We get the first notions of Good and Bad during our first years of life, when we learn how to behave in the environment and in the relationship with family and society, so the criteria learned in that period become the most powerful and durable during the life, constituting the fundamental structure of our decision system.

From some life experiences we extract positive conclusions, if the application of the final decision corresponded to our expectations, or negative conclusions, indicating that the theoretical decision did not match the practical situation. From each experience a conclusion is inferred, which can play a role as a decision criterion for the future experiences. And to complete the discussion on the role of decision criteria, let us remember again that the education during the first years of life (so called "the seven years from home") becomes defining for the personality of the later adult. The concepts of culture, faith, religion, everything which is included in the personal mentality, overlapping on the predispositions inherited from parents, fundamentally determine the thinking mode and the characteristic behavior of the future adult, translated from informational point of view by the formation and consolidation of the center Icd. If we also refer to the characteristic impulses received from GTS and MIS, reflected in the centers Ic and Ia respectively, we can understand that they can also play an important role in the defining a decision. MIS provides the body power by the feeding and by its absorption/desorption process, therefore the energy for its operation.

GTS plays the role as a species' continuity factor, mobilizing the body's resources not only for the reproduction, but also for the assurance of the survival and harmonious development of the next generation. Similarly, the center Ib can play a defining role, not only by the affiliation to a particular belief, but also by its stabilizing role for the body health, which we often instinctively appeal to, especially in the moments of life difficulty. We will therefore define the Attitude as an informational output of OIS (Operative Informational System), elaborated by the contribution of the entire information system of the organism [11]. Another important remark about Iw center: under the light of previous comments, it results that Iw can also be considered as a component of the Attitude. Indeed, depending on the concrete goal, not always what we truly want by Iw, it is really what we actually express through the Attitude. That is because at the elaboration of the Attitude, as we have pointed out above, participate all the other centers, by input of Information and by decision criteria. So, Iw remains one of the components, often the main, but not the unique component. We are therefore a complex information system, defined by seven informational centers, each of them with a distinctive, but interrelated functional role, working for the short and medium-term adaptation by means of OIS and for the species (long-term) survival by reproduction. We can therefore refer to the informational system of the organism as an integrated information system, resulting from the structural and functional co-participation of all its components: informational processors (systems), sensors, execution elements and informed-matter in general, as the information "hard". The attitude with respect to a certain criterion could be therefore positive (YES) or negative (NO). If our decisions with respect to the same criteria will conduct to a coincidence between the expectation and the results, then we will say that the selection criteria are "Good". However, if after repetitive attempts the result is not coincident, then we should conclude that the applied criteria are "Bad". A change it is necessary thus to operate within our thinking system. Any change in our thinking system, or any change in our living conditions, must be subjected to a fundamental rule: not to contravene to the natural laws of life, taking into account thereby the one's own life or that of the others.

In order to consciously decide our life way and therefore our destiny, we must therefore clarify toward what side we want actually to go on: that where we are going to defend the prerogatives of life, the health, keeping us therefore on its positive side, by conserving the joy of living, observing the gain and not the losses, the positive aspects and not the negative ones, or on a contrary side. For this, we have to start to observe the sort of information with we may accept or not. The selection of information which we have to operate with, is like that of food: "Good" or "Bad". A proper nutrition, ensuring an adequate functioning of the body, is selected from the vast existing variety, in order to be best suited to the current state of the body, reflected by la. Therefore, the proper selection of information, which is the "food" of the informational system, is equally important, providing the individual mental and corporal health. We have the possibility to choose by our free will: if we choose YES, a positive information, we will always find ourselves on the side that 
brings to our life thank, prosperity and joy, influencing in a defining way a positive destiny.

The choice between YES and NO does not only determines our long-term life trajectory, our destiny, but also our own health, which eventually definitely and definitively marks a happy destiny or not. The regular connection to $\mathrm{Ib}$ is always beneficial, bringing to the body the signals which it needs, that of encouragement, peace, confidence and optimism. However, the change of the thinking system is not an easy task, requiring a prolonged effort of self observing and control of the received and decisional information. As the attitude is a consequence of a sum of decision criteria coming from all informational centers, this should be carefully analyzed. Moreover, as it will be pointed out below, only repetitive cycles of experience goes to stabilize a new informational chain. This change is therefore a time and effort consumption mechanism, integrated in a reprogramming process of the thinking system. We have also to make a distinction between a controlled, conscious and progressive change process, and a spontaneous attitude change from YES to NO and reversely. When this pass from an innocent capricious game to a chronic, uncontrolled behavior mode to live, leading to a bipolar unstable personality, then serious consequences on the personal health could be Registered, as it will be discussed below. A stable and reliable balance between YES and NO should be therefore permanently maintained.

\section{Free will and the destiny}

The life cycle described in the Part I is the destiny of the species, the major coordinate of the life course, and there is no any possibility, at least for the moment, to be modified, only improved. This destiny applies naturally to every member of the species, and also to all the creatures on the Earth. The amount of information contained in the origin egg is sufficient to trigger the growth and the development of the fetus and that of the child, leading then the life of each individual, until his disappearance. But the genetic chains could intervene in our lives also in another manner, namely manifesting in Icd the personal specific predispositions, talents and skills. This is a gain that has to be detected and stimulated from very early age, as it is a "lesson" learned and transmitted by the previous generation or generations. By following the inner "call," once discovered the aptitudes, the distinct intrinsic attributes, it is much easier to traverse the professional training steps, because of the amount of initial information inherited from parental by genetic transmission and which is a privileged debut with respect to the competing partners in the same competition.

The start "in advance" provides therefore an easier and more successful trajectory. Inhibiting the skills of children for the sake of compelling to follow what the parents want insistently and authoritatively for their offspring, is a wrong attitude from this point of view. The Ic center of the parents, focused for the education and training of children, must also be adjusted in agreement with the reality. The discovery, stimulation and support of children' predispositions, skills and talents are not only recommended, but also necessary, to put the children on their own successful life path, because they benefit in this way of the informational "dowry" gained by the previous generation. Following such a trajectory, the destiny is predictable and is life easier to be driven. A more difficult situation is that when the life does not seem to smile us, or at least not as much as we would expect, so that the destiny seems to not help us. Two possibilities can be distinguished in this case, as shown below.

1. We are too exigent, we want too much, that is, the Iw center is super-active and unfitted to the reality; we will have two alternatives to choices in this case: to conform to reality, or to change the environment which we live in with other, corresponding to our aspirations.

2. We always follow the same path already used of a long time, the same way that made unhappy or dissatisfied our parents, our decisions being stereotyped, always or most often the same, declaring us to be the victims of our unhappy destiny. However, observing carefully what actually happens, we will need a major decision, that to change our mentality, be it inherited, or acquired through the parental education.

This decision is not easy to be applied, but becomes absolutely necessary, involving substitution of a series of habits and thinking stereotypes with new ones, able to lead us on a different path than the one of the Failure. It is therefore a matter of deciding the actual change of our system of values, criteria and mechanisms of thought. We can call this process a conscious remodeling or a reprogramming process, as will be explained in detail in a later dedicated book. Indeed, we cannot leave our own life at the hand of the random events, or destiny, it is necessary and justified our intelligent intervention in our own system of values through our free-will, to remedy the "defects" and to be able to benefit, by adaptation, to a new system of thinking, oriented towards a successful life and destiny. The maintenance of the emotional system II on the positive side is fundamental for the quality of life, because it represent actually the reaction of one's own body to the received information, so it affects or can affect the functioning of all the organs of the body. A sure way to poison our own existence is to "feed" ourselves with harmful, negative information. Any information that arouses or could arouse negative emotions is by definition negative.

We will live as captive in the life of our own choice: if our choice is always negative and we are prone to receive and operate with such kind of information, then our destiny will be always unsuccessful, and we will blame the "fate" as guilty. Worse than that, indulgent with ourselves, but not with the others, we will always see the others as responsible for our own failures or dissatisfactions and we will never find the right way of output from this vicious circle. The form under which the thinking and thus the associated negative emotions acts on the body is studied and reported as stress. By stress it is generally understood the pressure on the nervous system, deviating the organism from its normal, equilibrated functioning, but in a reversible way. However, if the stress is repetitive and of a long-time duration, this could affect irreversibly the normal functioning of organism, and it is translated into the body by reactions both of the brain itself, and of the body organs [12-14] (Figure 1). 


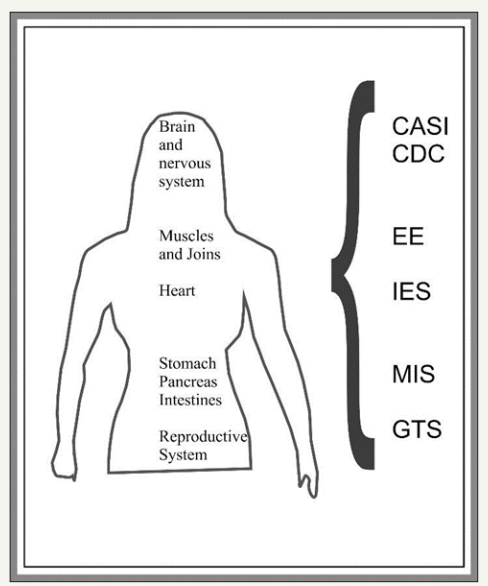

Figure 1: Body organs and systems affected by the application of the large-time periods of stress [13], triggered by the negatives repetitive thoughts (central side) and the corresponding informational/operational systems (right side), where EE represents the execution elements (muscles).

The dysfunctions of a normal mode of operation of the body organs are manifested starting with the simplest forms, such as the lack of appetite, apathy for any kind of activity, lack of energy, lack of sexual appetite, up to extreme forms. One of the most powerful effects is manifested on the brain and the nervous system by the installing and maintaining of the depression, the manic forms, anxiety and even schizophrenia $[12,14]$. Therefore, in order to assess whether the fate will prove to be attractive to us, or not, it is necessary to analyze the way in which we regard the life, by means of the two alternatives, YES or NO, available to the free will. So, it is good to be very careful with the information we acquire, and we memorize in CASI, becoming thereby the information source (Ik) for Iw. As we have commented, nature, as an informational system, both by its structural properties and by its laws, offers at the macro level two fundamental possibilities to decide, YES and NO. The YES part is the positive one, which protects the life, expressing its Associative character, the one which helps it, by the integration of information into matter to form and support living structures [1]. The love (II) is an associative property, manifesting itself by a Union force between a person, by means of his own informational system, and the object or the subject of love. From this point of view, the love has an anti-entropic, constructive character, helping the life and its prerogatives. The hate is the contrary of the love, having an entropic, disorder and destructive role, so does not help the prerogatives of life. As well as the hate, the envy, the jealousy, are destructive emotions, negatively affecting the own organism and that of the others, inducing long-term chronic diseases especially of the heart and of the circulatory system (palpitations, arrhythmias, coronary diseases, even the infarction and sudden death [15]), of the stomach, pancreas, intestines (digestive system in general (la)), of the muscles and joints (EE), of the reproductive system (Ic) $[13,14]$, as indicated in Figure 1. The respiratory system can also be affected by asthma $[13,14]$. Thus, the stress leads to release of histamine, which can trigger severe bronchoconstriction in asthmatics and respiratory disorder. The stress increases the risk of diabetes, particularly in overweight people, because the psychological stress changes insulin requirements [14]. The stress also alters the acid concentration in the stomach, which can lead to peptic ulcer, ulcers or ulcerative colitis and may increase the risk of prostate disease and the risk of severe reproductive system malfunctions in both women and men $[13,14]$. The contracting of chronic disorders can also be done indirectly by the affecting of the immune system.

The long-term effect of stress on the body's immune system is translated by two ways: (A) the weakening of it due to the brain's reaction by which it orders the production of cortisone with anti-inflammatory effect, reducing the action of the fighting agents and thus the defending efficiency against infections and colds; (B) the generation of autoimmune diseases such as rheumatoid arthritis, lupus or other autoimmune diseases that people recognize as inflammation, due to the excess of hormones transmitted as a response of the brain to the stress factors [12]. The mechanism by which the intoxication could occur, the poisoning of our own organism with negative information, is easy to be understand. Indeed, since the perception and storage of information in the brain is done by associating of the new information to the similar older one, already consolidated in the memory $[1,16]$, it results that once we ourselves have "contaminated" us with some negative obsessive thoughts, we will begin to absorb increasingly more, easier and easier, similar information to those which we already have been "furnished" our mental "scene", namely the negative one.

Through repetition, this growing thought congeries is assimilated and integrated into the automatic thinking system [3], which condemns us to be the prisoners of our own way of thinking. We become so dependent on the accumulated toxic information that was converted in our own way of living, and we no longer want (Iw) to detach from this whirlpool that descends us ever deeper to an inferior level of life. Acquiring and integrating the negative information into our informational system arrives to control our lives as a drug, and an effort of observation and will by means of Ia, Il and Iw is necessary to defeat this negative vortex where we are attracted to, harmful to the body and life. We will say that the life does not smile us, that the destiny is not favorable to us and that we have no luck in life, but in fact we are nothing more than the victims of our own negative thinking system. 
An absolute means of negative nervous poisoning is also the use of comparative examples, although apparently this would be harmless. Among the laws of memory is the fact that we memorize primarily the beginning and especially the end of a message. The vocal formulation of a message, whatever would be it, strengthens the chances of memorizing, while the repetition reinforces it. If we recourse to the method of contrast, trying to explain how good it is today, in relation to what was bad for example in the past, we will unfortunately remain in memory with priority with the last part of this message, the negative one. Therefore, instead to ignore the negative part, increasing the chances to be forgotten, we activate it and strengthen it in consciousness, keeping it as a toxic source of information. In this way, we will never be content actually with the present, and we will see the destiny as adversary, by means of the perpetual evocation of the negative past, even if the present is completely different from it. And as the present creates the foundations and perspective for the future, we will always remain tributary to the past, which we cannot separate from, even if we try (but in a wrong way) to reject it permanently. Another method of informational poisoning is the imagination of catastrophic scenarios, which could be evoked for instance as a contrast alternative even when everything was carried on a positive way, or of the personal image as a victim, as a "subscriber" ex officio to an unfair destiny. This manner to live the life prevents any manifestation of joy for the personal successes or that of the others. It seems strange, but yet this style is often practiced, especially by people who have probably lived some dramatic or traumatic situations in their childhood or during their youth and have become extremely "cautious" to not let be the "prey" of a sincere and natural joy. Certain people confuse the wisdom with the prohibition of enjoying their life, defying the benefits of this wonderful state of spirit, and taking on a severe "seriousness" role, in order to create an image of authority and pre-potency, especially with the passage of years and aging.

This style is also inadvisable, because it subjects the body to a permanent stress, forbidding it to a truly relax and recover of its consumed forces. And as information is acquired through association with the existing one, the followers of such a style will become the captives of their own thinking mode, seeking, selecting and accepting with absolute priority the accumulation of only the information that satisfy in a similar way those already acquired, i.e. the negative one. Therefore, they become some powerful "magnets" for the attraction of dramatic scenes, imagined or borrowed from others by today's abundant audio-visual means (especially through TV) and become its prisoners, willingly designing (but unfortunately in a wrong way), the negative destiny of their life. This kind of thinking leads to anxiety and depression. Joy is the most beautiful reward of fulfillment, as small as this could be, and fills and enriches the life, elevating its quality and prolonging it duration. It no must in any case be overshadowed, neither by the traumas borrowed or taken from the destinies of the others, and nor by the exacerbation of some imaginary dangers nowise.

The alternation between YES and NO works also in this case: after a period of dedication for achieving a goal, as small it would be, must be permitted to the body to enjoy of its fulfilment, conveying to it the gratitude for what it has done and Confidence in its forces for the future. The aggression, as a form of behavior, is also triggered and sustained by the obsessive negative thoughts, frequently operated and accepted as a form of a problem solving in the life. These thoughts generate impulses to violently attack, to hit someone, to harm or even kill a person, to Abuse of someone, to violently punish someone, or to say something rude, inappropriate and ugly to somebody [17]. An aggressive thought is a primary form of a future obsessive-compulsive disorder, because if at the beginning this seems to be only an innocent "imp" [18], but actually a young "devil", this can become by amplification and repetition a stereotype, a chronic and dangerous form of thinking, not only for himself or herself, but especially for those around. The aggressive thoughts, which become chronic, are psychotic and depressive forms to approach the life, requiring finally a proper medication [18]. The "solving" by aggression the differences with others is strictly forbidden, not only because it is a repugnant form to approach the inter-human relations, with grave and sometime irreparable consequences, but also because it can only lead to a mutual destruction.

Indeed, according to the law of action and reaction, applicable also in this case, it results that the injury/wound of a person returns by reaction against his or her own person sooner or later. This is a natural compensation law. The human species has reached the present performance level not by cultivating and exercising the brutal force, repelled, rejected and proved as inadequate by the history of humanity, but by the improvement of the informational system, developing it through experimentation and continuous adaptation, seeking and applying optimal solutions to each of the life problems in an intelligent way. If inherited, as it is the case for instance of the induced-depression risk [19], this thinking system needs to be modified by reprogramming. If it is a system acquired over the course of life, it must also be modified by the intervention of the free will, acting through Iw center. Otherwise, the effects of such kind of thinking can be manifested by mental affections [14], and if added the effect of the alcohol consumption, by severe disorders, including obsessive and manic behavior, deep depression, bipolar disorder of personality, aggressive behavior. Unbalancing the nervous system, otherwise functioning normally and consistently in a permanent and harmonious correlation with the environment and with itself, can therefore embrace extreme forms, becoming chronic in a long term, and which could be difficult to be treated even by specialized medical intervention.

Schizophrenia, a widespread disease in our modern society, manifested, among other things, by an uncontrolled mental reception of "voices", has recently been described as a form of nervous disorder by spontaneous "connection" to the information system of others [20]. From the point of view of the informational model described here, this connection is easy to be understood, because it can be done through the info-creational field of consciousness $[2,10]$. The bipolar attitude could be also reflected by an uncontrolled change between the extreme limits of YES and NO and reversely. Besides of a persistent negative thinking, this would be a 
consequence of a low control of mind and personal impulses, use of unreliable criteria and information, uncompensated stressing periods, high emotional/disproportional implication in the life events, some of the reasons discussed already above, or a combination of a part of the mentioned items, or of all of them. Therefore, a combined intervention plan should be applied to prevent the advance to a chronic disorder, including a reprogramming of the negative thinking operating system to a positive one.

The ways we can control the negative thinking and thus the stress as a preventative method or as an immediate action could be the following: (i) avoiding and disconnecting from the negative information sources; (ii) substitution of negative information with positive information; (iii) the mandatory observance of the resting and recovery by the sleeping during the night, because the nervous system also works according to YES (activated) and NO (disabled) alternations; (iv) the analysis of the motives that cause the negative thinking and their remediation [11]; (v) the participation at support groups [12,13], or to other alternatives [11], as a form of therapy; (vi) a regularly compensation of stress with physical and hobby activities, walks in nature, sports $[12,13]$, disabling thus the psyche from the focus of negative thoughts; (vii) the stimulation of the positive variant of life [21], by positive memories and positive reinterpretation of earlier older negative conclusions formulated during the past; (viii) the mental reprogramming by methods which will be presented in a future dedicated book; (ix) meditation and mental relaxation [12,18]; (x) addressing to professional counsels and applying a specialized medical treatment $[12,13]$. Besides the recommended, antiaging preventive methods cited above, but valid also to any age, a special attention should be paid to the amount of sleeping, which seems to be an independent risk factor of the mortality. A specific study dedicated to this issue [22], reports that the women who sleep $6-7$ hour per night show a minim mortality risk, while the women who sleep less than 5 hours or more than 9 hours, exhibit a significantly increased risk. So, besides the care on the alternation between the periods of the sleeping and of activity, necessary for the equilibration of the nervous system, it is necessary to apply an optimal rhythm and an optimal duration of the sleeping periods.

In relation with the above specified recommendations, a special additional reference could be done for a successful aging and life. A flexible adaptation, equilibrated balance between YES and NO is a helping and wise option for the older adults. The wisdom is, or should be, a specific quality during this stage, because of the entire life accumulated experience allows a appropriate selection between YES and NO. However, due to the rapid advance of the information technologies in our informatics era, a quick adaptation is necessary even at such seigniorial ages. The positive thinking is strongly recommended not only to prevent and recovery of some mild dysfunction and life prolongation, but also because this can consistently improve the quality and life expectancy, even in chronic terminal illnesses such as cancer [14]. Therefore, for their own benefit, it is strongly recommended that the older adults continue to learn, to be present and active in their day by day life, applying and promoting a positive and optimist thinking and feeling behavior in relation with themselves and with the others. This would be a favorable way to maintain a reliable communication with the younger generations, the family and the society in general, stimulating also the longevity and personal wellbeing.

\section{Conclusion}

We operate between two alternatives, YES and NO, and we have the possibility to choose by our free will one of them. To operate in a decisional system, it is necessary to dispose of decision criteria, the distinction between the "Good" (YES) and Bad (NO). The fundaments of the decision criteria are get by the child during the first years of life within the family, the parents transmitting to him their basic value system, characteristic for their culture, beliefs and social relations. As an extension of the genetic-related information received from the parents by IGG (Icd), the formation of the Icd center of the child is practically continued therefore by the direct info-transmission of specific parental life experience, which is integrated into his own criteria system. The attitude, as an info-reactive adaptation output of the informational system is a result mainly of the decisional operating system CDC (Iw), but all other informational centers (Ib, Ik, Il, Ia, Ic, Icd) can contribute with specific criteria to the final decision, especially the info-emotional one (II). According to our free will, it is possible to choose one of the two alternatives, YES or NO. By choosing the operating side (YES), preserving the life basic requirements and their stimulating characteristics like love, joy and especially a positive mode of thinking, we contribute to a positive destiny. A negative (NO) operating mode, can induce dysfunctions and sometimes serious disorders not only of the informational System itself, specifically of brain and the nervous cells, but also of the other organs and systems of the organism, like the digestive, immunity, circulatory and sexual system. The operating in a combined YES/NO bipolar mode is also undesirable, so the control of the info-emotional center (Il), the practicing of a positive thinking and of an equilibrated lifestyle is from this point of view a key to avoid the advance toward a chronic bipolar disorder. Some mechanisms contributing to the drop into a negative vortex were presented, based on the specific properties of the process of the information acquiring, consisting in a priority memorizing of that information which is of the same type with that already integrated in Ik by a repetition process. The using of a comparative analysis with the negative past, the catastrophic view of life, the victimization, the practice of negative emotions and thoughts like envy, jealousy, hate, are only a few of the starting platforms for a further large-scale proliferation of a negative thinking operational mode. The aggressive thoughts are dangerous even when these appeared as "innocent" newcomers, because by repetition they can be assimilated and integrated by the informational system. If a decision and/or some decisional criteria are proved to not correspond already with a satisfactory testing cycle, a reprogramming process of the thinking operational mode have to be applied. 
This should be however experienced progressively and under controlled conditions, the maintenance of the stability of the nervous system playing a primordial role during such a process. Therefore, under the circumstances presented above, the personal trajectory cannot be left to the hazard. The intervention of our free will is necessary for the decision to reprogram or to change our lifestyle, as well as for the selection of the input information, which it is negative, can intoxicate our informational system, condemning us to become the prisoners of our own negative thoughts, with evil consequences for the mental and body health. We can influence and design our own destiny by the practicing of a positive thinking mode, with beneficial effects on the mental and corporal health, on the improvement of the life quality and its prolongation. A successful life, aging and destiny are therefore the result of the personal lifestyle, based on a positive, self-controlled thinking system and on a permanent care to maintain it under performance conditions and suitable tolerance limits.

\section{Acknowledgment}

The author addresses his grateful thanks to the members of the editorial staff of this Journal, for their competent collaboration. To Adrian and Ana Maria and to Traian and Monica, for a beautiful and successful life.

\section{References}

1. Gaiseanu F (2017) Consciousness as informational system of the human body. Consciousness and Life Physics. Cosmology and Astrophysics Journal 16(1): 14-25.

2. Gaiseanu F (2017) Quantum-assisted process of disembody under near-death conditions: An informational-field support model. Neuro Quantology 15(1): 10-17.

3. Gaiseanu F (2017) An information based model of consciousness fully explaining the mind normal/paranormal properties. Neuro Quantology 15(2): 132-140.

4. Hajdukovic D (2010) Dark matter, dark energy and gravitational properties of antimatter.

5. Verlinde E (2010) On the origin of gravity and the laws of newton, Institute for Theoretical Physics University of Amsterdam Valckenierstraat 65 1018 XE, Amsterdam The Netherlands.

6. Carroll S (2015) The arrow of time and from eternity to here, the quest for the ultimate theory of time, GB: One world publication.

7. Fracasso C, Friedman H (2011) Near-death experiences and the possibility of disembodied consciousness: challenges to prevailing neurobiological and psychosocial theories. Neuro Quantology 9(1): 4153.

8. Gaiseanu F (2018) Near-death experiences and immortality from the perspective of an informational modeling of consciousness. Gerontology \& Geriatrics Studies 2(3): 1-3.

9. Meijer DKF (2013) Immortality: Myth or becoming reality? On the conservation of information. Syntropy 2013(3): 166-203.

10. Gaiseanu F (2018) An informational modeling of consciousness and cognitive centers, Proc. of the Human Project Brain (HPB) International Conference: Understanding Consciousness, A scientific quest for the $21^{\text {st }}$ century, 04-Models, simulation and emulation of consciousness, Barcelona, Spain.

11. Gaiseanu F, Graur A (2018) Cognitive centers related attitude: Application for an iterative evaluation method in music-based therapy process, Abstract Book of the Conference on Science of Consciousness (Co-Chair Prof. Stuart Hamer off, The University of Arizona-Center for Consciousness Studies, Tucson, Arizona), Consciousness and EducationCognitive Development, Concurent Session C27, 165: 2-7.

12. Krantz DS, Thorn B, Kiecolt Glaser J (2013) American Psychological Association (Revised 2013), How stress affects your health.

13. Wein H. Stress and disease: New perspectives.

14. Salleh MR (2008) Life event, stress and illness. Malays J Med Sci 15(4): 9-18.

15. Krantz DS, Whittaker KS, Sheps DS (2011) Psychosocial risk factors for coronary artery disease: Pathophysiologic mechanisms. In Heart and Mind: Evolution of Cardiac Psychology. Washington, USA.

16. Perlovski L (2001) Neural networks and intellect: Using model based concepts. Oxford: University Press.

17. Rachman S, de Silva P (1978) Abnormal and normal obsessions. Behav Res Ther 16(4): 233-248.

18. Baer L (2001) The Imp of the mind: Exploring the silent epidemic of obsessive bad thoughts. Dutton, New York, USA.

19. Goodman HG, Gotlib HI (1999) Risk for psychopathology in the children of depressed mothers: A developmental model for understanding mechanisms of transmission. Psychological Review 106(3): 458-490.

20. Beyer AC (2017) Theory of telepathy an alternative interpretation of psychotic experiences and some tips for recovery. Neuro Quantology 15(2): 145-159.

21. Davidson KW, Mostofsky E, Whang W (2010) Don't worry, be happy: Positive affect and reduced 10-year incident coronary heart disease: The Canadian Nova Scotia Health Survey. European Heart Journal 31(9): 1065-1070.

22. Patel SR, Ayas NT, Malhotra MR, White DP, Schernhammer ES, et al. (2004) A prospective study of sleep duration and mortality risk in women. Sleep 27(3): 440-444.

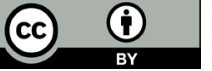

Creative Commons Attribution 4.0 International License

For possible submissions Click Here
Submit Article



\section{Gerontology \& Geriatrics Studies}

\section{Benefits of Publishing with us}

- High-level peer review and editorial services

- Freely accessible online immediately upon publication

- Authors retain the copyright to their work

- Licensing it under a Creative Commons license

- Visibility through different online platforms 